\title{
Foreword: programming language interference and dependence
}

\author{
DAVID CLARK ${ }^{\dagger}$, ROBERTO GIACOBAZZI and CHUNYAN MU \\ ${ }^{\dagger}$ Kings College, London, United Kingdom \\ Email: $\{$ David.J.Clark; Chunyan.Mu\}@kcl.ac.uk \\ ‡Università degli Studi di Verona, Verona, Italy \\ Email: roberto.giacobazzi@univr.it
}

Received 21 November 2010

Interference and dependence are closely related concepts: interference being the observable phenomenon connected with dependence. Essentially, interference means that the behaviour of some parts of a dynamic system may influence the behaviour of other parts of the same system, while dependence specifies how the semantics of sub-components of a dynamic system are related. Identifying, measuring and controlling interference is essential in many aspects of modern computer science, in particular, in security, program analysis and verification, debugging, systems specification, model checking, program manipulation, program slicing, reverse engineering, data mining, distributed databases and systems biology. In all these fields, dependency and interference play a key role in designing suitable abstractions or in partitioning complex systems into simpler ones. Reasoning about dependency and interference requires theories, models and semantics, as well as algorithms and tools for their analysis. Beginning in 2004, the series of Programming Language Interference and Dependence (PLID) workshops has been devoted to promoting and spreading cutting-edge research in this field, with a particular emphasis on unpublished results with great impact on the theoretical basis. PLID2007, which was held at the The Technical University of Denmark on 21 August 2007, was particularly successful, and constituted the ideal forum for announcing a call for papers for a special journal issue on programming language interference and dependence, which would not necessarily be restricted to PLID2007 contributions. From the many expressions of interest, we selected six contributions by leading researchers in the field, some of which had been presented at the PLID2007 workshop. The selected papers focus on foundational aspects of dependency and interference, with applications in language-based security, data-base management systems and program slicing.

The paper by Ana Almeida Matos and Jan Cederquist studies the information flows that occur in distributed programs in the presence of code mobility. This is a key problem in language-based security. The authors describe interesting new forms of security leaks that are introduced by code mobility, and which can be detected by means of a type and effect system for enforcing security, which is validated into an expressive calculus of mobile code.

The paper by Geoffrey Smith and Rafael Alpízar attacks the termination problem for security checking in the presence of random assignments. This is a difficult and important 
problem in language-based security and advanced mathematical models are required for its solution. The authors introduce an innovative notion of fast probabilistic simulation on Markov chains and show that this implies a key reachability property, which is essential in the checking of information flows. They go on to prove that well-typed probabilistic programs are guaranteed to satisfy an approximate probabilistic non-interference property, provided the probability of their non-termination is relatively small.

The paper by Gilles Barthe, Pedro R. D'Argenio and Tamara Rezk investigates logical formulations of secure information flow based on the idea of self-composition. This allows a practical and innovative reduction of the problem of secure information flow of a program $P$ to a safety property for a modified program, derived from $P$, by composing $P$ with a renaming of itself. This technique makes it easy to verify information flow policies by model checking and standard program verification methods, for example, in an $\grave{a}$ la Hoare logic.

The paper by Isabella Mastroeni and Anindya Banerjee explores the dimensions of a declassification-based non-interference policy, and provides a new perspective in this field. Two of the dimensions consist of specifying the power of the attacker, that is, what public information about a program an attacker can observe, and what secret information about the program needs to be protected. The third dimension, which regulates the above two, specifies the choice of program semantics. This choice can be formally specified as an abstract interpretation of a more concrete semantics.

The paper by James Cheney, Amal Ahmed and Umut A. Acar is a study of provenance, that is, the information recording the source or history of some computed data. The tracking of this information is particularly relevant in database management systems and security. The authors introduce an innovative formal semantics for modelling data provenance. Their idea is to use the notion of dependence, and corresponding models for dependence analysis and program slicing, for specifying provenance, thereby providing one of the first formal accounts of provenance in programming languages.

The paper by Sebastian Danicic, Robert M. Hierons and Michael R. Laurence explores the computational complexity of dynamic program slicing for schemas. The authors formalise Korel and Laski's definition of a dynamic slice as applied to linear schemas, and formulate an innovative and less restrictive definition in which the path through the original program need not be preserved by the slice. The problem of determining whether a given slice from a linear schema is a dynamic slice and whether a linear schema has a non-trivial dynamic slice is proved to be NP-hard. 